\title{
Modelo para la estimación de una frecuencia natural a partir de la respuesta vibratoria de un sistema sometido a un barrido sinusoidal de alta aceleración
}

\author{
A model to estimate a natural frequency based on the vibrational response \\ of a system excited by a high acceleration sine sweep \\ Ignacio Tomasov Silva ${ }^{1} \quad$ Cristian G. Rodríguez ${ }^{1 *}$ \\ Recibido 4 de marzo de 2016, aceptado 13 de marzo de 2017 \\ Received: March 4, 2016 Accepted: March 13, 2017
}

\begin{abstract}
RESUMEN
Una forma de detectar una frecuencia natural de una maquina o estructura es a partir de la respuesta vibratoria debido a una excitación de frecuencia variable. Cuando la frecuencia de la excitación se acerca a una frecuencia natural, se produce un aumento en la amplitud vibratoria. Si la tasa con la que varía la frecuencia de la excitación es alta en relación a la frecuencia natural, entonces la máxima amplitud vibratoria se produce cuando la frecuencia de la excitación es distinta a la frecuencia natural. Esta diferencia puede inducir a errores al momento de determinar una frecuencia natural durante una partida o parada de una máquina. En este artículo se evalúa la diferencia entre las frecuencias de máxima amplitud vibratoria y natural de un sistema de un grado de libertad, y se propone un modelo para obtener la frecuencia natural a partir de la frecuencia de máxima amplitud vibratoria. Se demuestra, por medio de resultados experimentales obtenidos a través de ensayos sobre una viga empotrada, que el valor de una frecuencia natural estimada a partir del modelo propuesto es una mejor aproximación que el obtenido al estimar dicha frecuencia natural como la frecuencia de excitación en la que ocurre la máxima respuesta vibratoria.
\end{abstract}

Palabras clave: Resonancia, excitación no estacionaria, barrido sinusoidal, frecuencia natural, alta aceleración, respuesta vibratoria, modelo.

\section{ABSTRACT}

A natural frequency from a machine or structure can be determined from the vibratory response due to a variable frequency excitation. There is a rise in vibratory amplitudes at the moment the frequency of excitation is close to natural frequency. If the change rate in the excitation frequency is high in comparison to natural frequency, then the maximum vibration amplitude will occur when the excitation frequency is different to natural frequency. This difference could induce errors in the determination of natural frequencies during startup and shutdown. In this article we assess the difference between natural frequency and maximum amplitude frequency of a simple one degree of freedom system, and a model is proposed to obtain natural frequency from maximum amplitude frequency. It is concluded, by means of experimental results obtained in tests on a cantilever beam, that the value of a natural frequency estimated from the proposed model is a better approximation than that obtained when the natural frequency is estimated as the frequency of excitation when the maximum vibratory response occurs.

Keywords: Resonance, non-stationary excitation, sine sweep, natural frequency, high acceleration, vibration response, model.

1 Laboratorio de Vibraciones Mecánicas. Departamento de Ingeniería Mecánica. Universidad de Concepción. Edmundo Larenas 270 interior. Concepción, Chile. E-mail: cristian.rodriguez@udec.cl; ignatomasov@udec.cl

* Autor correspondencia. 


\section{INTRODUCCIÓN}

Una forma de estimar frecuencias naturales de una maquina o estructura, consiste en medir la amplitud de la vibración que experimenta dicho sistema mientras se aplica una excitación de frecuencia variable o barrido sinusoidal. Se asume que el valor de las frecuencias naturales detectadas es igual al valor de las frecuencias de excitación a las que se experimentan las máximas amplitudes de vibración. No obstante, este método permite obtener solamente una aproximación del valor real de una frecuencia natural, ya que no considera las alteraciones que experimenta la respuesta vibratoria cuando la frecuencia de la excitación varía rápidamente en el tiempo (variaciones mayores a un $1 \%$ de la frecuencia natural por segundo). Cuando la aceleración del barrido utilizado es extremadamente baja, la envolvente de la respuesta vibratoria coincide con la respuesta estacionaria del sistema. Por el contrario, cuando la aceleración del barrido es alta, la máxima amplitud de vibración obtenida es menor que la amplitud máxima de la respuesta estacionaria y ocurre cuando la fuerza excitadora tiene una frecuencia diferente al valor de la frecuencia natural [1]. Para limitar estas alteraciones la norma ISO 7626 sugiere un valor límite de aceleración a emplear en barridos sinusoidales utilizados para determinar frecuencias naturales [2].

La complejidad en el desarrollo de un modelo que prediga la respuesta vibratoria ante un barrido sinusoidal, recae en la incapacidad de solucionar de forma analítica la ecuación diferencial que modela dicho fenómeno. Varios autores han contribuido en el desarrollo de aproximaciones teóricas o soluciones numéricas para resolver dicha ecuación diferencial [3-7]. Markert [7], propone una solución analítica aproximada, entregando ecuaciones que estiman la máxima amplitud vibratoria y la frecuencia del barrido en la que ocurre la amplitud máxima de la vibración. Sin embargo su modelo no considera el efecto de las condiciones iniciales del barrido, las cuales, como se presentará en este artículo, generan variaciones significativas en el valor de la frecuencia de máxima amplitud de la respuesta vibratoria.

En este trabajo, se presenta un modelo para la estimación de la frecuencia natural de un sistema, a partir de la respuesta vibratoria ante barridos sinusoidales de mediana-altas tasas de aceleración.
Se realiza una comparación del método propuesto con mediciones experimentales, demostrándose que el valor de una frecuencia natural obtenido a partir del modelo propuesto, es una mejor aproximación que el obtenido al estimar dicha frecuencia natural, como el valor de la frecuencia de excitación en el que ocurre la máxima amplitud de respuesta vibratoria.

\section{RESPUESTA ESTACIONARIA DE UN SISTEMA SDOF}

Se utiliza la respuesta estacionaria de un sistema de un grado de libertad SDOF (single degree of freedom) como referencia para medir las alteraciones de la vibración ante un barrido sinusoidal. La respuesta estacionaria del sistema con masa $m$, amortiguamiento $c$ y rigidez $k$, sometido a una fuerza sinusoidal de frecuencia $\Omega$ de amplitud máxima $F_{o}$ es:

$$
x_{e s t}(t)=X_{o} \sin \left(\Omega t+\phi_{x}\right)
$$

Donde, $X_{o}$ es la amplitud de la respuesta estacionaria dada por la ecuación (2), y $\phi_{X}$ es el desfase entre la respuesta vibratoria y la fuerza excitatoria, relacionadas mediante la ecuación (3).

$$
\begin{gathered}
X_{o}(\Omega)=\frac{F_{o}}{\sqrt{1-\left(\frac{\Omega}{\omega_{n}}\right)^{2}+\left(\frac{2 \xi \Omega}{\omega_{n}}\right)^{2}}} \\
\tan \left(\phi_{x}-\phi_{F}\right)=\frac{\frac{2 \xi \Omega}{\omega_{n}}}{1-\left(\frac{\Omega}{\omega_{n}}\right)^{2}}
\end{gathered}
$$

En las ecuaciones (2) y (3), $\xi$ es el factor de amortiguamiento, y $\omega_{x}$ es la frecuencia natural del sistema. La fase $\phi_{F}$ corresponde al ángulo inicial del vector oscilatorio de fuerza. Para el caso estacionario, la frecuencia para la cual se obtiene la máxima amplitud de respuesta vibratoria es:

$$
\max \left(X_{o}(\Omega)\right)=\omega_{n} \sqrt{1-2 \xi^{2}} \approx \omega_{n}
$$

Dado que el factor de amortiguamiento es comúnmente bajo (típicamente menor a 0,02 ), la 
frecuencia en la que se obtiene la máxima amplitud estacionaria se puede suponer igual al valor de la frecuencia natural del sistema, según se muestra en la ecuación (4).

\section{ECUACIÓN DEL MOVIMIENTO DE UN SISTEMA SOMETIDO A UN BARRIDO SINUSOIDAL DE TASA CONSTANTE}

Un barrido sinusoidal de tasa constante es una excitación oscilatoria en la cual la frecuencia $\Omega(t)$ de la fuerza excitadora $F(t)$ cambia a una tasa constante en el tiempo:

$$
\Omega(t)=a t+\omega_{s}
$$

Donde $\alpha$ corresponde a la tasa con que varía la excitación, y $\omega_{s}$ es la frecuencia inicial de la fuerza excitadora. La fuerza $F(t)$ puede definirse como una excitación sinusoidal de amplitud máxima constante, con una variación de frecuencia dada según la ecuación. (5), obteniéndose la siguiente función:

$F(t)=F_{o} \sin \left(\int_{0}^{t} \Omega(t) d t\right)=F_{o} \sin \left(\frac{a t^{2}}{2}+\omega_{s} t+\beta\right)(6)$

Donde $\beta$ corresponde a la fase de las excitaciones o posición angular inicial de la excitación, análogo a $\phi_{F}$ en (3). La ecuación del movimiento que modela la respuesta vibratoria de un sistema SDOF, ante una excitación de barrido sinusoidal de amplitud constante, es:

$$
m \ddot{x}+c \dot{x}+k x=F_{o} \sin \left(\frac{a t^{2}}{2}+\omega_{s} t+\beta\right)
$$

La ecuación (7) puede ser reescrita en función de su frecuencia natural $\omega_{n}$ y de su factor de amortiguamiento $\xi$ :

$$
\ddot{x}+2 \xi \omega_{n} \dot{x}+\omega_{n}^{2} x=\left(\frac{F_{o}}{m}\right) \sin \left(\frac{a t^{2}}{2}+\omega_{s} t+\beta\right)
$$

Con el objetivo de analizar cómo los parámetros de la ecuación (8) afectan en la respuesta vibratoria, se define el tiempo normalizado $\tau$ :

$$
\tau=t \cdot \omega_{n}
$$

Las funciones derivadas y dependientes de $t$, serán dependientes de $\tau$ a través de la regla de la cadena. Se define la aceleración normalizada o tasa normalizada de aceleración $\alpha$, y la velocidad inicial normalizada $\eta_{s}$.

$$
\begin{gathered}
\alpha=\alpha / \omega_{n}^{2} \\
\eta_{s}=\omega_{s} / \omega_{n}
\end{gathered}
$$

De este modo la ecuación diferencial planteada en la ecuación (8), expresada en función del tiempo normalizado $\tau$, y con las sustituciones previamente descritas, es:

$$
\frac{d^{2} x}{d \tau^{z}}+2 \xi \frac{d x}{d \tau}+x=\left(\frac{F_{o}}{m \omega_{n}^{z}}\right) \sin \left(\frac{a \tau^{2}}{2}+\eta_{s} \tau+\beta\right)
$$

Esta ecuación puede ser resuelta mediante la integral de convolución, para esto es necesario definir la respuesta impulsional $h(\tau)$ del sistema, y precisar la excitación $F(\tau)$ a la cual este está sometido. Estas funciones son:

$$
\begin{gathered}
h(\tau)=\frac{e^{-\xi \tau}}{\sqrt{1-\xi^{2}}} \sin \left(\tau \sqrt{1-\xi^{2}}\right) \\
F(\tau)=\left(\frac{F_{o}}{m \omega_{n}^{2}}\right) \sin \left(\frac{\alpha \tau^{2}}{2}+\eta_{s} \tau+\beta\right)
\end{gathered}
$$

La respuesta del sistema, expresada mediante la integral de convolución, queda definida según:

$$
\begin{gathered}
x(\tau)=\left(\frac{F_{o}}{m \omega_{n}^{2}}\right) \int_{0}^{\lambda} \sin \left(\frac{\alpha \lambda^{2}}{2}+\eta_{s} \tau+\beta\right) * \\
\frac{e^{\xi(\lambda-\tau)}}{\sqrt{1-\xi^{2}}} \sin \left((\tau-\lambda) \sqrt{1-\xi^{2}}\right) d \lambda
\end{gathered}
$$

La ecuación previamente descrita no tiene solución analítica debido a que no existe una solución teórica 
para el término $\int \sin \left(\lambda^{2}\right) d \lambda$. Por este motivo, para evaluar los resultados y posteriormente compararlos con los resultados experimentales, se resolverá la integral de convolución de forma numérica.

\section{RESPUESTA ANALÍTICA APROXIMADA}

Markert [7] propone una solución aproximada para la respuesta vibratoria ante un barrido sinusoidal de tasa constante [6]. Esta se basa en un desarrollo analítico en conjunto a una evaluación numérica de la función error compleja. Dentro de sus alcances permite calcular la envolvente de la respuesta vibratoria, la que omitiendo ciertos términos, esta descrita por la expresión:

$$
x_{e n v}(\Omega)=|B \cdot w(v)| \text {. }
$$

Donde $w$ es la llamada función error compleja:

$$
w(v)=e^{-v^{2}}\left(1+\frac{2 i}{\sqrt{\pi}} \int_{0}^{v} e^{u 2} d u\right)
$$

$v$ es una función compleja en función del tiempo normalizado:

$$
v(\tau)=-\frac{1+i}{2 \sqrt{\alpha}}\left(\alpha \tau+\eta_{s}-\xi i-\sqrt{1-\xi^{2}}\right)
$$

y $B$ es una constante compleja:

$$
B=\frac{1-i}{4 \sqrt{\alpha}} \sqrt{\frac{\pi}{\alpha}}\left(\frac{F_{o}}{k}\right)
$$

La función error compleja, necesaria para determinar la respuesta vibratoria, debe ser evaluada de forma numérica ya que el término $\int_{0}^{v} e^{u 2} d u$ no posee solución analítica. El desarrollo previamente presentado no considera el efecto de la fase inicial de las excitaciones $\beta$.

\section{ALTERACIONES DE LA RESPUESTA VIBRATORIA}

La función frecuencia de excitación $\Omega(t)$, puede utilizarse como variable independiente para graficar la amplitud de la respuesta vibratoria, esto permite compararla con la respuesta estacionaria del sistema $x_{o}(\Omega)$.

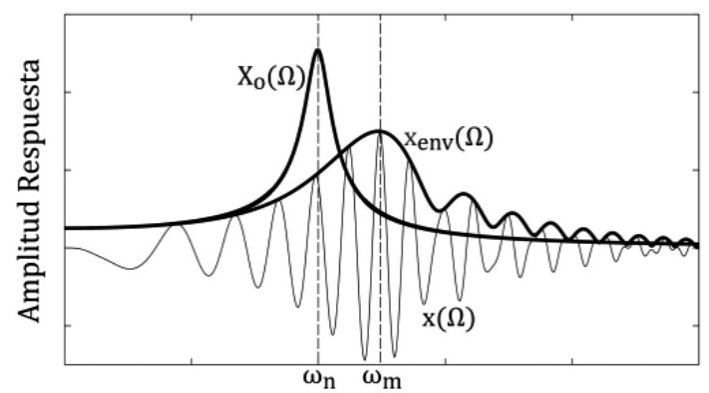

Frecuencia de excitación $\Omega$

Figura 1. Comparación de la respuesta vibratoria del sistema $x(\Omega)$ y su envolvente con la respuesta estacionaria $x_{o}(\Omega)$.

Se observa de la Figura 1 que la envolvente de la respuesta vibratoria $x_{e n v}(\Omega)$, tiene una forma diferente a la respuesta estacionaria del sistema, dichas diferencias son las llamadas alteraciones de la respuesta vibratoria. Estas se caracterizan por tener una menor amplitud máxima respecto a la respuesta estacionaria, y por experimentar dicha amplitud máxima en una frecuencia diferente a la frecuencia natural. También es característico de la respuesta vibratoria ante un barrido sinusoidal, la aparición de máximos locales de vibración tras el paso por una frecuencia natural. Estos pueden ser interpretados como "beats" producto de la suma de dos vibraciones con frecuencias muy cercanas, una a la frecuencia de la excitación, y otra a la frecuencia natural del sistema.

Para estudiar de forma cuantitativa las alteraciones que se producen en la respuesta vibratoria debido a un barrido sinusoidal de alta tasa, se define la frecuencia en la cual se experimenta la máxima amplitud de respuesta vibratoria como $\omega_{m}$. Para independizar la frecuencia de excitación $\Omega$, de la frecuencia natural del sistema, se define la frecuencia normalizada de excitación $\eta$. Este corresponde al valor de la frecuencia de la excitación dividido por la frecuencia natural del sistema.

$$
\eta=\Omega / \omega_{n}
$$

Del mismo modo, es posible normalizar el valor de $\omega_{m}$ respecto al valor de la frecuencia natural del sistema.

$$
\eta_{R}=\omega_{m} / \omega_{n}
$$


Esta normalización permite cuantificar la desviación que presenta la frecuencia de amplitud máxima, independiente del valor de la frecuencia natural.

\section{MODELOS PARA ESTIMACION DE LA FRECUENCIA DE AMPLITUD MÁXIMA}

Conocer la amplitud máxima que puede experimentar la respuesta vibratoria de un rotor durante la partida o parada es fundamental en el diseño y la operación de estos. Es por esto que varios autores han propuesto aproximaciones para la respuesta vibratoria de un sistema lineal SDOF sometido a una excitación de barrido sinusoidal que pasa por una zona resonante [3-7]. Al entregar expresiones aproximadas para la amplitud máxima de la respuesta vibratoria, también ofrecen aproximaciones para predecir la frecuencia en la que ocurre dicha amplitud máxima. Se compararán algunas de las aproximaciones para la estimación de $\eta_{R}$.

Leul [6] propone una aproximación analítica basada en un método de variación de parámetros.

$$
\eta_{R}=1+\operatorname{sign} \alpha \frac{2.222 \sqrt{|\alpha|}}{1+0.556 \xi / \sqrt{|\alpha|}}
$$

Markert [7] obtiene analíticamente una función aproximada que relaciona la frecuencia en la cual ocurre la máxima amplitud de la respuesta vibratoria con la tasa normalizada de las excitaciones.

$$
\eta_{R}=1+2.157 \operatorname{sing} \alpha \sqrt{|\alpha|}
$$

Notar que ambos modelos relacionan un único valor de $\eta_{R}$ según la tasa normalizada de aceleración $\alpha$ utilizada en la excitación. Esto se debe a que en las deducciones utilizadas para determinar dichos modelos desprecian el efecto de las condiciones iniciales $\eta_{s}$ y $\beta$, en pro de enfatizar la influencia de la tasa normalizada $\alpha$ sobre la amplitud de la respuesta del sistema.

La Figura 2 presenta la amplitud de la respuesta vibratoria según las ecuaciones (16) y (23) para un barrido con tasa positiva y tasa negativa:

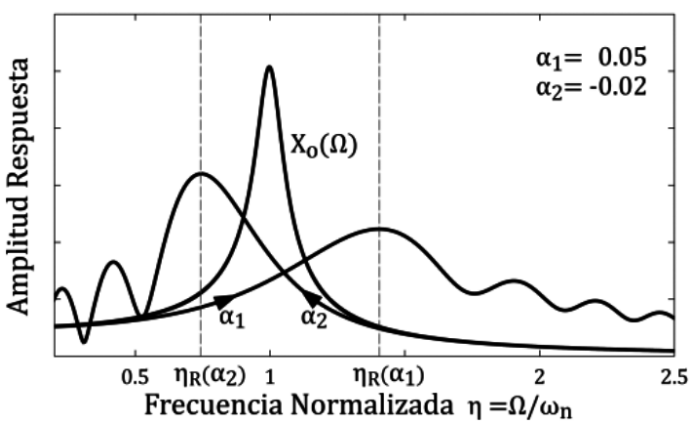

Figura 2. Influencia de la aceleración normalizada $\alpha$ sobre el valor $\eta_{R}$

Notar que para valores de $\alpha$ positivos el valor de $\eta_{R}$ es mayor que 1 , mientras que para valores de $\alpha$ negativos, de $\eta_{R}$ es menor que 1 .

\section{INFLUENCIA DE LAS CONDICIONES INICIALES DEL BARRIDO SINUSOIDAL}

Las condiciones iniciales del barrido corresponden al valor $\eta_{s}$, y al valor $\beta$ presentado en la ecuación (15). Al calcular la respuesta numérica se observa que distintos valores de condición inicial producen distintos valores de $\eta_{R}$ cuando se aplica un barrido con una aceleración $\alpha$ fijo. Los distintos valores de $\eta_{R}$ obtenidos quedan contenidos en un rango de posibles valores, de modo que, cualquier condición inicial elegida produce una respuesta vibratoria con un valor de $\eta_{R}$ dentro de dicho rango. Este rango puede ser caracterizado mediante un valor medio $\eta_{R \text {, alt. }}$ y un valor alterno $\eta_{R \text {, alt. }}$ Es decir, para una misma tasa de barrido sinusoidal, existen múltiples posibles frecuencias de máxima amplitud debido a que distintas fases implican distinta forma de excitar el sistema al momento que la frecuencia de la excitación se acerca a la frecuencia natural.

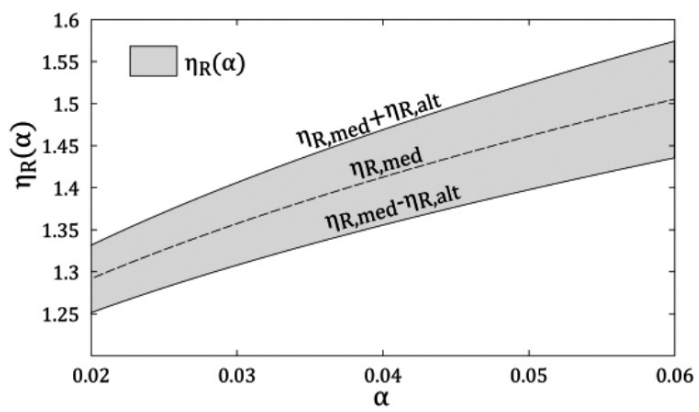

Figura 3. $\eta_{R}$ en función de $\alpha$. Se destacan las funciones $\eta_{R, \text { med }} \mathrm{y} \eta_{R \text {, alt }}$. 
Notar que para un barrido sinusoidal con una tasa de excitación $\alpha$ en específico, y un determinado rango de variación de las condiciones iniciales $\eta_{s}$ y $\beta$, existe un único valor de $\eta_{R, \text { med }} \mathrm{y} \eta_{R, \text { alt }}$. De este modo es posible encontrar un modelo que ajuste los valores de $\eta_{R \text {, med }} \mathrm{y} \eta_{R, \text { alt }}$ en función de la tasa de las excitaciones $\alpha$.

\section{MODELO PARA $\eta_{R, \text { med }} \mathbf{y} \eta_{R, \text { alt }}$}

Se obtiene el rango $\eta_{R, \text { med }} \mathrm{y} \eta_{R, \text { alt }}$ a partir de la solución numérica de la integral de convolución definida en la ecuación (15). Esta es resuelta considerando un rango de las condiciones iniciales $\eta_{s}$ y $\beta$, para cada valor tasa de aceleración $\alpha$ evaluada. El resultado de la evaluación numérica es ajustado a un modelo matemático, el cual cumple con la evaluación del caso estacionario $\eta_{R, \text { med }}(\alpha=0)=1$ y con la razón de proporcionalidad observada por Markert [7] entre $\backslash \mid \alpha$ y $\eta_{R}$. Este modelo (Tomasov 2016) se presenta en las ecuaciones (24) y (25):

$$
\begin{gathered}
\eta_{R, \text { med }}=1+2.057 \operatorname{sing}(\alpha) \sqrt{|\alpha|} \\
\eta_{R, \text { alt }}=0.413 \sqrt{|\alpha|}
\end{gathered}
$$

Los valores $\eta_{R, \text { med }} \mathrm{y} \eta_{R \text {, alt }}$ pueden ser representados según la definición dada por la ecuación (21):

$$
\begin{aligned}
& \eta_{R, \text { med }}=\omega_{m, \text { med } /} \omega_{n} \\
& \eta_{R, \text { alt }}=\omega_{m, \text { alt }} / \omega_{n}
\end{aligned}
$$

De este modo, siendo conocido el valor de $\omega_{m, \text { med }} \mathrm{o}$ $\omega_{m \text {, alt }}$, y el valor de la tasa $\alpha$ aplicada en el barrido sinusoidal, es posible determinar un rango para el valor real de la frecuencia natural por medio del modelo propuesto.

La comparación de los modelos propuestos por Markert y Leul, con el modelo propuesto en esta investigación se grafica en la Figura 4.

Se observa en la Figura 4 que, tanto el modelo propuesto por Markert como el modelo Propuesto por Leul, quedan contenidos dentro del modelo propuesto en esta investigación.

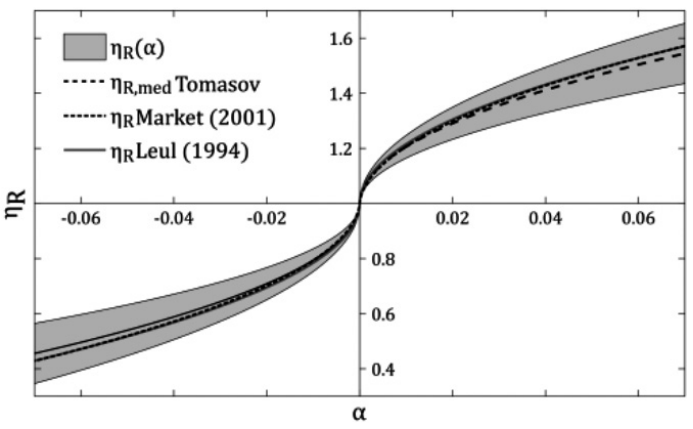

Figura 4. $\eta_{R \text {, med }} \mathrm{y} \eta_{R \text {, alt }}$ según modelo propuesto por Tomasov, respecto a los modelos propuestos por Markert y Leul.

\section{COMPARACIÓN DEL MODELO PROPUESTO CON RESULTADOS EXPERIMENTALES}

Con el fin de evaluar el modelo propuesto, se diseña un banco de ensayos para medir la respuesta vibratoria de un sistema con frecuencias naturales conocidas, ante una excitación de barrido sinusoidal con tasa de excitaciones y condiciones iniciales del barrido controlables.

El sistema implementado consiste en una viga empotrada, excitada en un extremo mediante un Shaker modelo TIRAvib s 51110. Se asume que el primer modo de vibrar de la viga unida al shaker tiene un comportamiento como el modelado para un sistema lineal de un grado de libertad.

La señal de excitación es creada mediante el software Labview y generada mediante la tarjeta NI 6711, luego es amplificada en elequipo TIRA BAA 120 de modo que la amplitud de la corriente suministrada al shaker, y por tanto, la fuerza oscilatoria ejercida por este sobre el sistema sea de magnitud constante. Tanto las aceleraciones como la fuerza excitadora se registran en el extremo de la viga, y son adquiridas mediante una tarjeta NI 9233. Un transductor modelo PCB 208 B02 es utilizado para verificar las fuerzas aplicadas por el excitador. Éste une un extremo de la viga al shaker mediante un cable tenso, el cual se mantiene siempre en tracción en el transcurso de los ensayos. El excitador y la viga se presentan en la Figura 5.

Los parámetros modales del sistema implementado se registran mediante un análisis modal experimental por medio de un ensayo de golpe. Este es llevado 


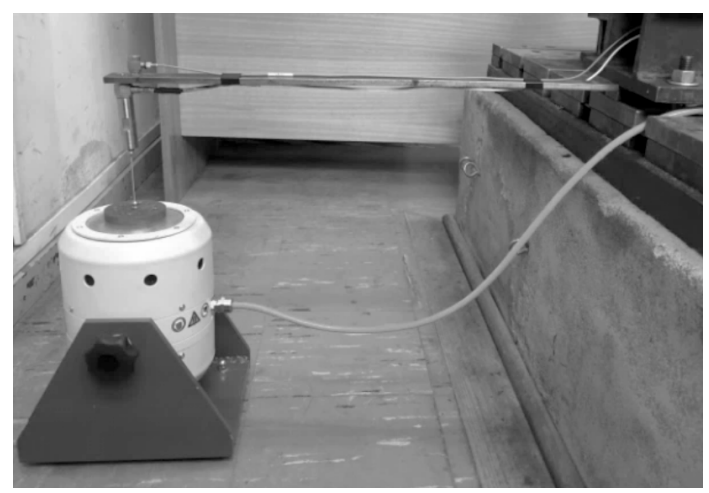

Figura 5. Banco de ensayos: viga empotrada excitada mediante un shaker.

a cabo con un martillo Brüel \& Kjær 8206, dicha fuerza excitadora se registra en conjunto con la aceleración mediante la tarjeta NI9233. Tanto el punto de excitación como de medición se escoge en el extremo de la viga. Dichos parámetros modales son verificados antes, durante, y al terminar la ronda de ensayos de barridos sinusoidales. Estos no presentaron variación en los parámetros modales determinados durante el trascurso de dichos ensayos.

Las funciones respuesta obtenidas por ensayo de golpe son promediadas y procesadas mediante el algoritmo Least squares complex exponential [8], el cual permite encontrar en las frecuencias naturales y amortiguamientos que minimizan el error respecto a los datos medidos. Se obtiene que la primera frecuencia natural del sistema tiene un valor $\omega_{1}=23.63 \mathrm{~Hz}$ y un factor de amortiguamiento $\xi_{1}=1.36 \%$. Se verifica además que los parámetros modales del sistema no varían cuando el shaker se encuentra encendido o apagado.

Se determina experimentalmente el área de valores posibles $\eta_{R}$ en función de $\alpha$. Para esto se registra la respuesta vibratoria de la viga ante barridos sinusoidales con diferentes tasas normalizada $\alpha$. Cada tasa $\alpha$ es ensayada para diferentes valores de la fase de excitaciones $\beta$ entre 0 y $180^{\circ}$. Cada barrido de tasa $\alpha$ positiva es realizado con una velocidad inicial $\eta s=0.09$, mientras que los de tasa $\alpha$ negativa con $\eta s=2.31$.

Se obtienen los valores $\eta_{R}$ experimentales según la ecuación (21), utilizando la frecuencia de amplitud máxima $\omega_{m}$ medida ante cada barrido sinusoidal, y el valor de la frecuencia natural $\omega_{1}$ determinada a través del análisis modal experimental. Los resultados experimentales obtenidos son comparados con el modelo propuesto en la Figura 6.

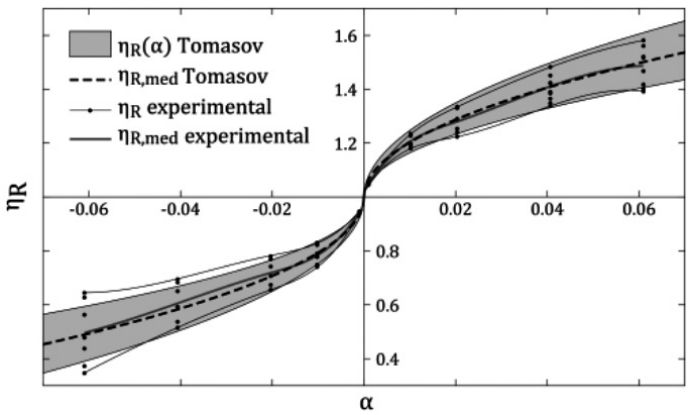

Figura 6. Comparación de $\eta_{R}$ experimental vs $\eta_{R}$ predicho por modelo propuesto.

Del mismo modo puede ser estimado el valor de la primera frecuencia natural del sistema $\omega_{1}$ a partir de los valores experimentales $\omega_{m}$ y el modelo matemático planteado para $\omega_{m \text {, med }}$. Como cada excitación aplicada para una tasa $\alpha$ tiene una fase inicial $\beta$ diferente, es posible encontrar el valor $\omega_{m \text {, med }}$ de los valores $\omega_{m}$ registrados, de modo que junto al modelo propuesto permitan despejar el valor de $\omega_{1}$ como:

$$
\omega_{1}=\omega_{m, \text { med }} / \eta_{R, \text { med }}
$$

Una vez obtenida la estimación del valor de la frecuencia natural mediante la ecuación (28), se calcula el error relativo de ésta respecto al valor real de la frecuencia natural obtenida experimentalmente. Dicho error es comparado al error relativo asociado a asumir que la frecuencia natural corresponde al valor de la frecuencia en cual ocurre la amplitud máxima $\omega_{m}$.

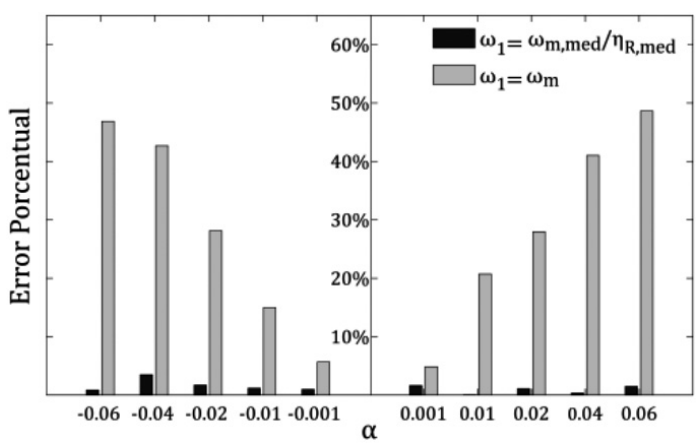

Figura 7. Comparación del error en la determinación de la frecuencia natural $\omega_{1}$ para cada tasa de excitación ensayada. 
Se observa de la Figura 7 que la estimación de $\omega_{t}$ basada en la utilización del modelo propuesto, tiene una menor desviación respecto al valor real que el estimar la frecuencia natural como la frecuencia de excitación en el cual ocurre la máxima amplitud de vibración $\left(\omega_{m}\right)$. Sin embargo, para poder utilizar el modelo propuesto, es necesario contar con un conjunto de respuestas vibratorias ante barridos con una misma tasa $\alpha$, cada una con distintas condiciones iniciales. Esto es una desventaja, ya que en la práctica no es posible conocer las condiciones iniciales del barrido sinusoidal. Por otro lado, el método propuesto permite estimar la frecuencia natural del sistema cuando la aceleración de las excitaciones es alta, y la respuesta vibratoria es de corta duración, condición que impide obtener la frecuencia natural mediante otros métodos de procesamiento de señales [9-10].

Cabe destacar, que el error de la frecuencia natural estimado como la frecuencia de excitación en el cual ocurre la máxima amplitud vibratoria, disminuye en la medida que la magnitud de la tasa de excitación $\alpha$ utilizada es menor. Este error, puede ser estimado a priori utilizando las ecuaciones (24) y (25). De este modo es posible elegir la aceleración de un barrido en función del error tolerable en la determinación de la frecuencia natural.

\section{CONCLUSIONES}

La estimación del valor de una frecuencia natural a partir del modelo propuesto, es una mejor aproximación que el valor obtenido al estimar dicha frecuencia natural como la frecuencia de excitación en el cual ocurre la máxima amplitud de respuesta vibratoria, cuando la aceleración del barrido empleado es de mediano o alto valor.

La incorporación de las condiciones iniciales del barrido sinusoidal permite obtener una mejor aproximación de la frecuencia en la cual ocurre la amplitud máxima de un sistema sometido a un barrido sinusoidal que los métodos actualmente presentes en la literatura.

\section{AGRADECIMIENTOS}

Se agradece al Laboratorio de Vibraciones Mecánicas de la Universidad de Concepción. Su aporte humano y técnico permitió el desarrollo de esta publicación.

\section{REFERENCIAS}

[1] C. Lalanne. "Mechanical Vibration and Shock Analysis". ISTE. Vol. 1. London, UK. 2009. ISBN: 0863802184.

[2] ISO. Vibration and Shock: Experimental Determination of Mechanical Mobility, Parts 1-5, iSO-7626/1-5.

[3] F.M. Lewis. "Vibration during acceleration through a critical speed". Journal of Applied Mechanics. Vol. 54, pp. 253-261. 1932.

[4] G. Hok. "Response of linear resonant systems to excitation of a frequency varying linearly with time". Journal of Applied Physics. Vol. 19, pp. 242-250. 1948.

[5] R.V. Trull. "Sweep speed effects in resonant systems". The Shock and Vibration Bulletin. Vol. 41, Part 4, pp. 95-98. December, 1970.

[6] F. Leul. "Zum transienten Schwingungsverhalten beim Resonanzdurchgang linearer Systeme mit langsam zeitveränderlichen Parametern". Bericht 4d Instituts für Mechanik der Universitä-GH Kassel. Germany, pp. 207- 217. 1994.

[7] R. Markert. "Analytically based estimation of the maximum amplitude during passage through resonance", International Journal of Solids and Structures. Vol. 38, Issues 10-13, pp. 1975-1992. Marzo, 2001. ISSN: 0020-7683. DOI: $10.1016 / \mathrm{S} 0020-7683$ (00)00147-5.

[8] D. Ewins. "Modal Testing: Theory, Practice and Application". Research Studies Press Ltd. 2nd Edition. Somerset, UK.2000. ISBN: 0863802184.

[9] S. Junichi and M. Dias. "Modal analysis of structures under non-stationary excitation". Engineering Structures. Vol. 99, pp. 56-62. September, 2015. ISSN: 0141-0296. DOI: 10.1016/j.engstruct.2015.04.035.

[10] G. Gloth and M. Sinapius. "Analysis of swept-sine runs during modal identification". Mechanical Systems and Signal Processing. Vol. 18, Issues 6, pp. 1421-1441. November, 2004. ISSN: 0888-3270.doi:10.1016/ S0888-3270(03)00087-6. 